\title{
The ubiquitin ligase Rnf6 regulates local LIM kinase 1 levels in axonal growth cones
}

\author{
Baris Tursun, ${ }^{1,5}$ Anne Schlüter, ${ }^{1,5}$ Marvin A. Peters, ${ }^{1}$ Birte Viehweger, ${ }^{1}$ Heather P. Ostendorff, ${ }^{1}$ \\ Juliana Soosairajah, ${ }^{2}$ Alexander Drung, ${ }^{1,3}$ Michael Bossenz, ${ }^{1}$ Steven A. Johnsen, ${ }^{1}$ \\ Michaela Schweizer, ${ }^{1}$ Ora Bernard, ${ }^{2}$ and Ingolf Bach $^{1,3,4,6}$ \\ ${ }^{1}$ Zentrum für Molekulare Neurobiologie Hamburg (ZMNH), Universität Hamburg, 20251 Hamburg, Germany; ${ }^{2}$ The Walter \\ and Eliza Hall Institute for Medical Research, Victoria 3050, Australia; ${ }^{3}$ Program in Gene Function \& Expression, ${ }^{4}$ Program \\ in Molecular Medicine, University of Massachusetts Medical School, Worcester, Massachusetts 01605-2324, USA
}

\begin{abstract}
LIM kinase 1 (LIMK1) controls important cellular functions such as morphogenesis, cell motility, tumor cell metastasis, development of neuronal projections, and growth cone actin dynamics. We have investigated the role of the RING finger protein Rnf6 during neuronal development and detected high Rnf6 protein levels in developing axonal projections of motor and DRG neurons during mouse embryogenesis as well as cultured hippocampal neurons. RNAi-mediated knock-down experiments in primary hippocampal neurons identified Rnf6 as a regulator of axon outgrowth. Consistent with a role in axonal growth, we found that Rnf6 binds to, polyubiquitinates, and targets LIMK1 for proteasomal degradation in growth cones of primary hippocampal neurons. Rnf6 is functionally linked to LIMK1 during the development of axons, as the changes in axon outgrowth induced by up- or down-regulation of Rnf6 levels can be restored by modulation of LIMK1 expression. Thus, these results assign a specific role for Rnf6 in the control of cellular LIMK1 concentrations and indicate a new function for the ubiquitin/proteasome system in regulating local growth cone actin dynamics.
\end{abstract}

[Keywords: LIM kinase; actin cytoskeleton; axon development; primary hippocampal neurons; protein stability; ubiquitin; proteasome]

Received June 6, 2005; revised version accepted August 10, 2005.

For the establishment of neuronal circuits, axons of developing neurons are directed by guidance cues to their correct destinations. The growth cone is located at the tip of the outgrowing axon and senses guidance cues in the environment and translates this information into changes in the cytoskeleton, thereby determining the direction of growth (Sanes and Lichtman 1999; Dickson 2002). Ubiquitination events are crucial for the development and functions of neurite outgrowth and neuronal circuits (Murphey and Godenschwege 2002; DiAntonio and Hicke 2004; van Horck et al. 2004). However, little is known as to the identities of the molecules involved in these processes.

LIM kinase 1 (LIMK1) is located predominantly in the cytoplasm and serves as a critical regulator of the cellular actin cytoskeleton. Activation of the Rho/Rock or Rac/PAK signaling pathways results in the phosphorylation and activation of LIMK1, thereby enabling it to phosphorylate the actin depolymerizing factors ADF/co-

\footnotetext{
${ }^{5}$ These authors contributed equally to this work.

${ }^{6}$ Corresponding author.

E-MAIL Ingolf.Bach@umassmed.edu; FAX (508) 856-4650.

Article and publication are at http://www.genesdev.org/cgi/doi/10.1101/ gad.1340605
}

filin. Phosphorylation of cofilin leads to an increase in actin polymer (F-actin) formation by decreasing the ability of cofilin to bind to and depolymerize actin (Arber et al. 1998; Yang et al. 1998). It is thought that this ability to influence the dynamics of actin polymerization is responsible for the various cellular functions that have been attributed to LIMK1. These functions range from the regulation of cell morphogenesis and motility, to tumor cell metastasis, to the development of neuronal projections. In neurons, LIMK1 has been shown to be involved in the regulation of neuronal growth cone actin dynamics and axon formation as well as the development of hippocampal dentritic spines, synapses, and long-term potentiation (LTP) (Sarmiere and Bamburg 2002; Fukazawa et al. 2003; Gungabissoon and Bamburg 2003; Rosso et al. 2004). Changes in LIMK1 concentration have been found to be important for most of these functions.

The covalent attachment of ubiquitin to its target protein occurs in a series of enzymatic steps that are mediated by the ubiquitin-activating enzyme (E1), ubiquitinconjugating enzyme (E2), and the ubiquitin ligase (E3). The ubiquitin ligase determines the specificity of this reaction by forming protein-protein interactions with 
the E2 enzyme and the substrate protein. Polyubiquitinated substrate proteins are often targeted for degradation by the $26 \mathrm{~S}$ proteasome. The RING finger has been identified as a conserved protein domain harboring the catalytic domain of the ubiquitin ligases (Pickart 2001). The ubiquitin ligase RLIM regulates the activity of LIM homeodomain proteins (LIM-HD), a class of transcription factors that plays important roles for the development of neuronal structures and cell types (Bach 2000; Hobert and Westphal 2000; Shirasaki and Pfaff 2002). RLIM mediates polyubiquitination of CLIM cofactors (cofactors of LIM-HD, also known as NLI, Ldb), which are required by LIM-HD proteins to exert their developmental activity (Becker et al. 2002; Thaler et al. 2002; Matthews and Visvader 2003), thereby targeting them for proteasomal degradation (Bach et al. 1999; Ostendorff et al. 2002; Hiratani et al. 2003). Here, we identify the RING finger protein Rnf6, belonging to a family of RLIM-like proteins, as an ubiquitin ligase, able to bind to and mediate polyubiquitination of LIMK1. We show that Rnf6 protein is highly expressed in projections of neuronal cell types, where LIMK1 is localized, and present evidence that Rnf6 targets LIMK1 for proteasomal degradation in axonal growth cones of primary hippocampal neurons, with functional implications for axonal outgrowth. Thus, our results indicate an Rnf6-mediated regulation of growth cone actin dynamics via LIMK1 levels.

\section{Results}

Identification and expression of the RLIM-like RING finger protein family

Mammalian LIM-HD proteins are subdivided into groups based on sequence conservation with two representatives for each group (Hobert and Westphal 2000). Since there are also two CLIM cofactors in mammals (Bach et al. 1997), we predicted the existence of a second, RLIM-like molecule. A search of the GenBank database identified Rnf6 as the protein with the highest sequence homology to RLIM. In particular, functional domains such as the RING finger and the basic domain displayed high sequence homologies.

In order to gain insight into the similarities and divergences of Rnf6 and RLIM, we raised specific antibodies and compared the distribution of both proteins during mouse neural tube development. We focused these studies on the neural tube since the biological activity of several LIM homeodomain proteins, in conjunction with CLIM cofactors, is involved in the correct development of axonal projections of specific neurons in the ventral neural tube (Shirasaki and Pfaff 2002). Indeed, we detected expression of both Rnf6 and RLIM proteins in the ventral portion of the developing neural tube of embryonic days 12.5-13 (E12.5-E13) mouse embryos (Fig. $1 \mathrm{~A}, \mathrm{~B} \mid$, coinciding with expression of LIM-HD proteins in this region (Shirasaki and Pfaff 2002). Furthermore, both proteins were highly expressed in the floorplate (Fig. $1 \mathrm{~A}, \mathrm{~B})$. Surprisingly, higher magnification revealed a dif-

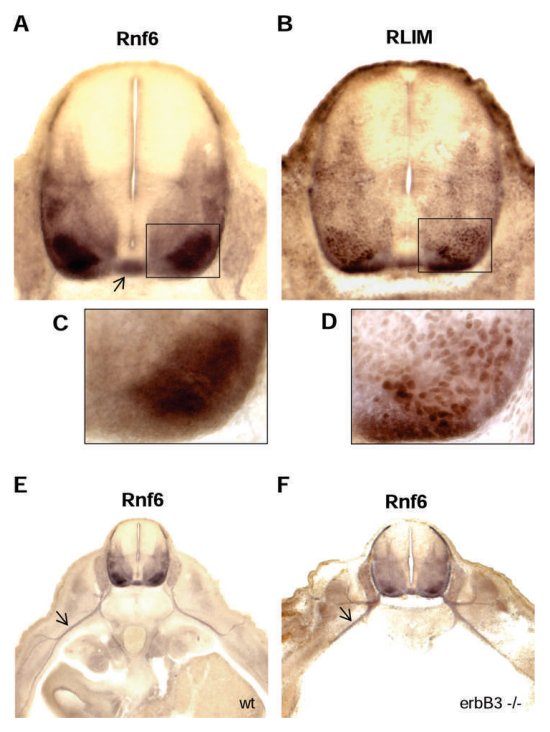

Figure 1. Rnf6 and RLIM proteins are differentially expressed in E12.5-E13 mouse spinal cord. Unless otherwise indicated, transversal vibratome sections at the thoracic level of wild-type mice are shown. Immunohistochemical staining of E12.5-E13 mouse embryos using anti-Rnf6 $(A)$ and anti-RLIM $(B)$ polyclonal antibodies. High Rnf6 and RLIM expression is observed in similar regions of the developing ventral neural tube where motor- and inter-neurons reside and the floorplate (arrow). $(C)$ Diffused Rnf6 staining in the developing mouse embryo indicating cytoplasmic expression. Higher magnification of the region boxed in $A$. (D) RLIM is highly expressed in nuclei of cells during mouse neural tube development. Higher magnification of the region boxed in $B$. Rnf6 staining in putative axonal projections of E12.5-E13 wild-type $(E)$ and erbB3 ${ }^{--}(F)$ mouse embryos. The arrows indicate staining of axonal projections.

fused Rnf6 expression pattern, indicating cytoplasmic expression (Fig. 1C,D). We also detected Rnf6 in long neuronal extensions resembling axonal projections of DRG and ventral neural tube neurons (Fig. 1E). To identify whether these structures were axonal projections or glial cells accompanying these projections, we stained sections of erbB3-null mice, that at E12.5 temporarily display axonal projections but lack glial cells (Riethmacher et al. 1997), with our Rnf6 antibody. The detection of Rnf6 protein in sections of erbB3-null mouse embryos indicated that Rnf6 is expressed in axonal projections (Fig. 1F).

\section{Rnf6 regulates axon outgrowth in cultured primary hippocampal neurons}

One established model system to study neurite development is the primary culture of dissociated hippocampal neurons (Dotti et al. 1988; Craig and Banker 1994). Cultured neurons first extend several undifferentiated neurites (stages 1 and 2). At stage 3, one of these neurites grows rapidly and becomes the axon, whereas the other neurites subsequently develop into dentrites at stage 4 . 
Since we found Rnf6 expression in developing neuronal projections during mouse embryogenesis, we examined its distribution in primary hippocampal neurons prepared from newborn mice (post-natal day 1 [P1]) at stage 3 using our rabbit anti-Rnf6 polyclonal antibodies. It is thought that axons develop at this stage, leading to a polarization of neurons (Craig and Banker 1994). As expected, we found that Rnf6 is widely expressed in the cytoplasm and projections of stage 3 neurons (Fig. 2A). We detected high levels of Rnf6 in growth cones of projections that appeared as developing axons, suggested by their increased lengths and high levels of the axonal marker Tau. A similar expression pattern has been reported for the neuronal polarity proteins mPar3/mPar6 (Shi et al. 2003). Next, we investigated the effects of Rnf6 down-regulation on cultured hippocampal neurons using siRNA oligonucleotides. Only specific Rnf6 siRNA significantly reduced the level of the endogenous Rnf6 protein but not that of the related RLIM protein (Fig. 2B) in cell extracts prepared from siRNA-transfected neurons. Cotransfection of this siRNA with the GFP-C3 expression construct dramatically increased the axonal length of the transfected neurons in comparison with neurons cotransfected with GFP-C3 alone and control siRNA (Fig. 2C). While the average axonal length of neurons transfected with control siRNA was $\sim 85 \mu \mathrm{m}$, that of neurons transfected with Rnf6 siRNA was almost twice as long $(\sim 170 \mu \mathrm{m})$ (Fig. 2D). Expression of Rnf6 siRNA had no effect on the number of neurites per neuron.

These results suggest that Rnf6 is important for the control of axon outgrowth as its down-regulation results in a significant increase in the length of axons of cultured primary neurons $24 \mathrm{~h}$ after transfection. We therefore overexpressed GFP-Rnf6 in cultured neurons and observed a significant decrease in neurite length, from $\sim 90$ $\mu \mathrm{m}$ for control-transfected neurons to $\sim 30 \mu \mathrm{m}$ (Fig. 2E,F). RING-finger-deleted versions of E3 ligases often behave as dominant-negative molecules (Pickart 2001). There-
A

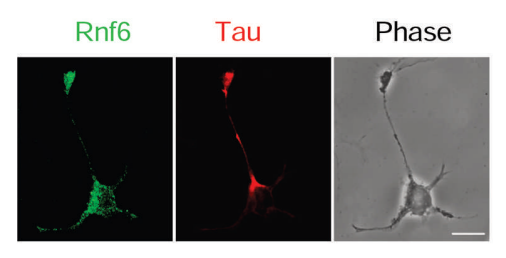

C

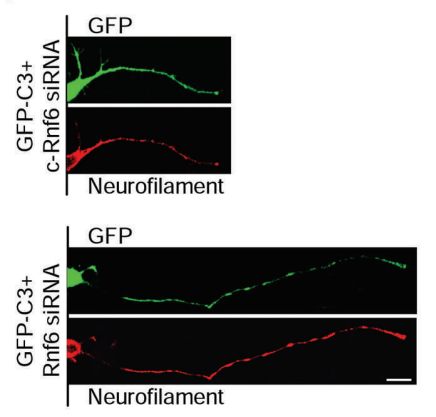

$\mathbf{E}$

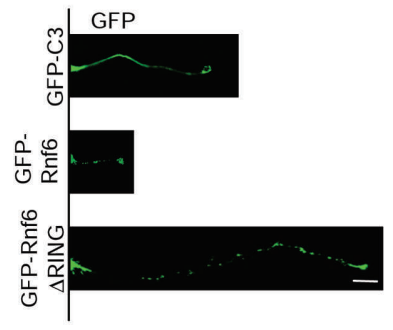

B

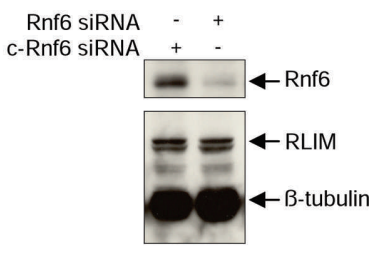

D

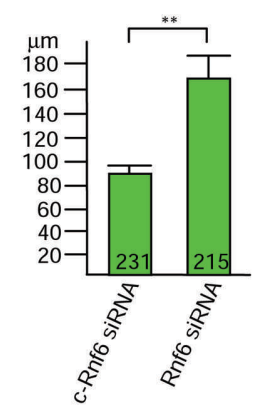

$\mathbf{F}$

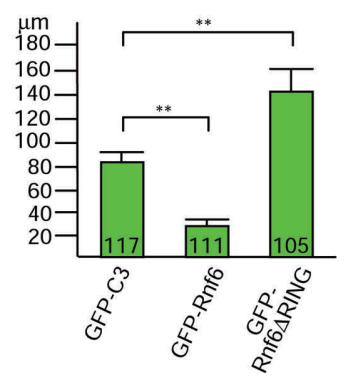

Figure 2. Rnf6 regulates axonal growth in primary hippocampal neurons. (A) Rnf6 is expressed in growth cones of primary hippocampal neurons. Neurons were cultured for $36 \mathrm{~h}$ and stained with Rnf6 antibodies (green) and antibodies directed against the axonal marker Tau (red). Note strong Rnf6 staining in the growth cone of the projection with the highest Tau levels, which will most likely develop into the axon. (Right panel) A phase-contrast image of the same neuron. Bar, $10 \mu \mathrm{m} .(B)$ Treatment of primary hippocampal neuron cultures with Rnf6 siRNA decreases the level of Rnf6 protein. Western blot of protein extracts of Rnf6 siRNA and control siRNA (c-Rnf6 siRNA) transfected primary hippocampal neuron cultures probed with specific Rnf6 as well as with RLIM and $\beta$-tubulin antibodies as specificity and loading controls, respectively. (C) Down-regulation of Rnf6 in primary hippocampal neurons results in increased axon length. Primary neurons were cultured for $24 \mathrm{~h}$ prior to cotransfection of siRNAs with the empty pEGFP-C3 vector to visualize transfected cells. Twenty-four hours later, cells were processed for immunocytochemistry and stained with anti-neurofilament antibody. GFP signals (green, upper panels) and neurofilament staining (red, lower panels) of representative projections are shown. Bar, $10 \mu \mathrm{m}$. (D) Statistical analysis of axon lengths of Rnf6 siRNAtransfected neurons. The mean values of three independent transfections of control siRNA and Rnf6 siRNA are shown. The total number of neurons measured is indicated for each transfected siRNA. Neurofilamentpositive projections were measured using analySIS Pro software. $\left(^{\star \star}\right) P<0.0001$. Error bars indicate SEM. (E) Analysis of the axon lengths of primary hippocampal neurons transfected with constructs expressing GFPRnf6, GFP-Rnf6 RING, and GFP-C3. Neurofilamentpositive projections were measured, using analySIS Pro software. Representative neuronal projections of transfected cells are shown. Bar, $10 \mu \mathrm{m}$. (F) Statistical analysis of axon lengths of neurons transfected with GFP fusion constructs. The mean values of three independent transfections are shown. The total number of neurons measured is indicated for each transfected construct. $\left(^{\star \star}\right) P<0.0001$. Error bars indicate SEM. 
Tursun et al.

fore we also examined the effects of GFP-Rnf6 6 RING expression on axonal length of transfected neurons. Consistent with a role of Rnf6 for neurite development, the RING-finger-deleted Rnf6 protein functioned in a dominant-negative manner and led to an increase in axonal length of the transfected neurons in a similar way to that of neurons treated with RNF6 siRNA (Fig. 2E,F). In contrast, overexpression of RLIM had no significant effect on neurite length (data not shown).

\section{Rnf6 interacts with and targets LIMK1 for proteasomal degradation}

Because of the great similarity between RLIM and Rnf6, and since the effects of Rnf6 on neurons is dependent on its RING finger (Fig. 2E,F), we postulated that Rnf6, like RLIM, is also an E3 ubiquitin ligase targeting proteins for degradation. It is therefore possible that $\mathrm{Rnf} 6$ regulates axonal outgrowth indirectly by mediating targeted proteasomal degradation.

We have previously shown that LIMK1 can bind to RLIM (Bach et al. 1999). Due to the high conservation of the LIM-domain-interacting basic domain between RLIM and Rnf6, we examined the possibility that Rnf6 also interacts with LIMK1. Indeed, in GST pulldown assays, Rnf6 and RLIM interacted with both mouse and rat LIMK1 (Fig. 3A). In coimmunoprecipitations of Cos 7 cell lysates coexpressing HA-tagged LIMK1 and the more stable RING-finger-deleted Myc-Rnf6 (Myc-Rnf6 $\mathrm{RING)}$ and Myc-RLIM (Myc-RLIM $\Delta$ RING), both proteins interacted with HA-LIMK1 (Fig. 3B, lanes $5,8)$. To show interaction in primary hippocampal neurons, and to rule out the possibility that the previously observed interaction between these two proteins was due to the large amount of proteins in the overexpression system, we performed coimmunoprecipitation of the endogenous proteins. Immunoprecipitation using primary hippocampal cell lysates with anti-LIMK1 mAb or nonspecific IgG followed by Western blotting with anti-Rnf6 antibodies demonstrated an interaction between endogenous Rnf6 and LIMK1 (Fig. 3C).

RING finger proteins are ubiquitin ligases, able to autoubiquitinate (Lorick et al. 1999). Therefore, we first confirmed that Rnf6 is, indeed, an ubiquitin ligase, able to autoubiquitinate in a RING-finger-dependent manner in the presence of the $\mathrm{E} 2$ enzyme $\mathrm{UbcH} 5$, by performing in vitro ubiquitination assays (Fig. 4A). Because Rnf6 and RLIM can interact with LIMK1, we investigated whether LIMK1 could serve as a substrate for both ubiquitin ligases. Intriguingly, both E3s were able to mediate ubiquitination of LIMK1 (Fig. 4B). Most or all lysine residues (K) on an ubiquitin molecule can form ubiquitin-ubiquitin isopeptide linkages, and the type of linkage greatly influences the fate of the ubiquitinated protein. A protein can be modified at one or more lysine residues by a single ubiquitin molecule (monoubiquitination), by lysine-linked chains of ubiquitin (polyubiquitination), or combinations of both. Proteins attached to K48-linked polyubiquitin chains are degraded by the $26 \mathrm{~S}$ proteasome (Weissman 2001). To distinguish between these possibilities, we performed in vitro ubiquitination assays with LIMK1 using Rnf6 as E3 ligase and ubiquitin mutant proteins lacking all lysine residues (K0) or containing only one lysine at position 48 (K48only). Whereas the assays with the K0 ubiquitin resulted only in a weak ubiquitination of LIMK1 representing monoubiquitina-
Figure 3. Rnf6 associates with LIMK1 in cells. (A) Rnf6 and RLIM interact with LIMK1 in vitro. (Upper panel) ${ }^{35}$ S-labeled in vitro translated full-length mouse and rat LIMK1 (mLIMK1 and rLIMK1) proteins were tested for their ability to interact with GST-Rnf6 and GST-RLIM fusion proteins. (Lower panel) As input control, half of the reaction was run in parallel on a separate gel and proteins were visualized by Coomassie blue staining. $(B)$ Overexpressed Rnf6 and RLIM interact with overexpressed LIMK1 in cells. Cell extracts of HeLa cells transfected with Myc- and HA-tagged constructs were immunoprecipitated with the indicated antibodies. To obtain higher protein levels, we used Rnf6 and RLIM constructs with deleted RING fingers. The Western Blot was probed with anti-HA antibodies. For negative controls, cells were cotransfected with HA-LIMK1 and the empty Myc vector pCS2-MT, which expresses six Myc epitops, or with the nonrelated Myc-Lhx3 expression plasmid. The arrow indicates the band expected for HA-LIMK1. (C) Interaction of endogenous Rnf6 and LIMK1 in primary hippocampal neuron cultures. Total cell extracts of primary hippocampal neurons, treated with the proteasome inhibitor MG132 for $6 \mathrm{~h}$, were immunoprecipitated with either anti-LIMK1 mAb or nonspecific IgGs. The Western blot was probed with anti-Rnf6 or, as control, anti-LIMK1 antibodies. Twenty percent input is shown. The arrows indicate the bands expected for Rnf6 or LIMK1.
A
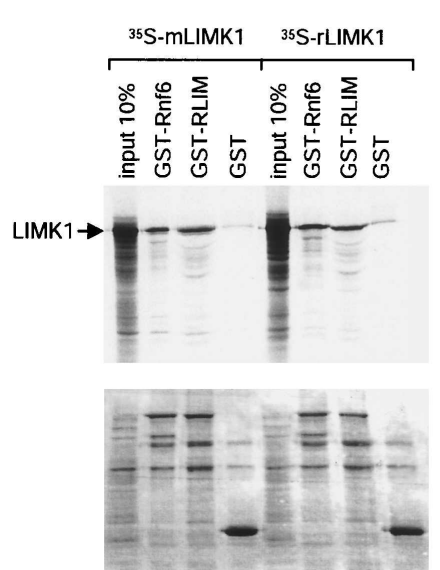

Protein Input
B

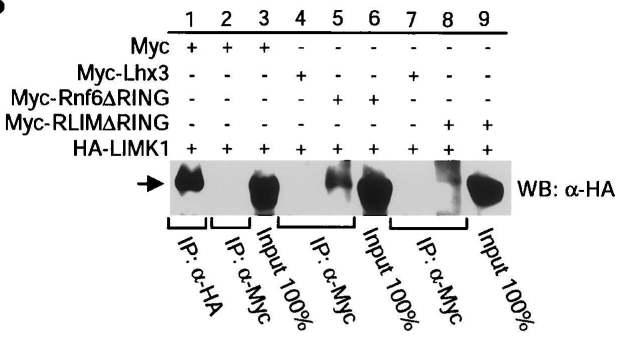

C

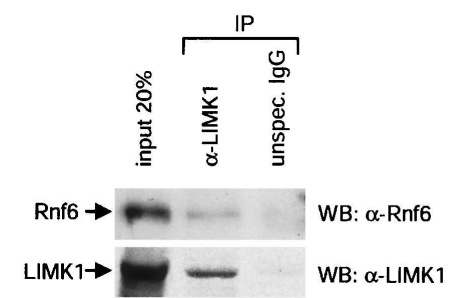




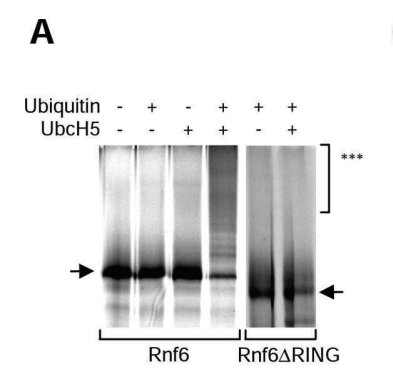

C

B

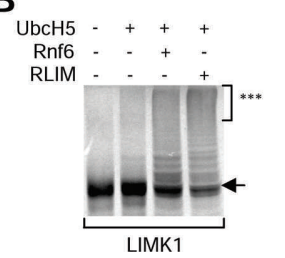

E
Ubiquitink48only -
Ubiquitin

Ubiquitinko - - + - .

Rnf6 - + + + +

$\mathrm{UbcH} 5+-+++$
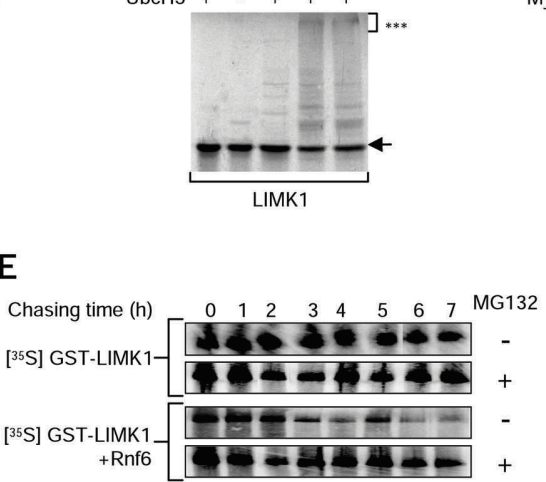

D

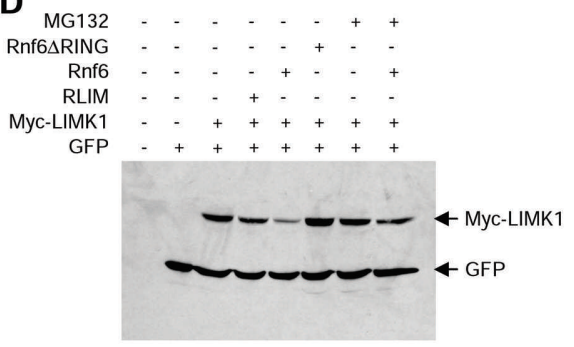

F

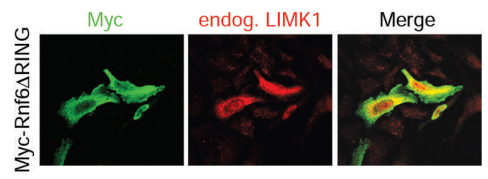

Figure 4. Rnf6 polyubiquitinates and targets LIMK1 for proteasomal degradation. (A) Rnf6 is a RING-finger-dependent ubiquitin ligase. In vitro ${ }^{35} \mathrm{~S}$-labeled full-length Rnf6 protein or the RING-finger-deletion mutant Rnf6 $\Delta \mathrm{RING}(\Delta 589-667)$ protein was incubated with or without the ubiquitin-conjugating (E2) protein UbcH5. The arrow indicates the position of the nonubiquitinated Rnf6 proteins. Polyubiquitinated proteins are indicated by three asterisks $\left(^{\star \star \star}\right)$. Note that in contrast to full-length Rnf6, the RING-finger-deletion mutant cannot autoubiquinate. (B) Bacterially expressed Rnf6 and RLIM mediate ubiquitination of ${ }^{35}$ S-labeled LIMK1 protein in vitro. The arrow indicates nonubiquitinated LIMK1 protein. Polyubiquitinated proteins are indicated by three asterisks $\left({ }^{\star \star \star}\right)$. $(C)$ Rnf6 polyubiquitinates LIMK1, generating K48 isopeptide linkages. Wild-type (wt) ubiquitin, ubiquitin lacking all lysine residues (K0), or an ubiquitin containing only one lysine at position 48 (K48only) was used for the in vitro ubiquitination assays. The arrow indicates nonubiquitinated LIMK1 protein. Polyubiquitinated proteins are indicated by three asterisks $\left(^{\star \star \star}\right)$. Note that incubation with the K0 ubiquitin mutant protein results in little LIMK1 ubiquitination, whereas ubiquitination reactions using the K48only ubiquitin mutant protein were almost as efficient as those using wild-type ubiquitin. $(D)$ Rnf6 targets LIMK1 protein for degradation. Western blots of total protein extracts of HEK293T cells cotransfected with Myc-LIMK1 and untagged RLIM, Rnf6, or the deletion mutant Rnf6 RING constructs were probed with anti-Myc antibodies. $(E)$ Rnf6 decreases the half-life of LIMK1. GST-LIMK1 was expressed in Cos7 with or without Rnf6, and the proteins were labeled with ${ }^{35} \mathrm{~S}$-methionine for $1 \mathrm{~h}$, followed by washings and chasing in normal medium for time periods up to $7 \mathrm{~h}$ in the presence or absence of MG132. At each time point, GST-LIMK1 was pulled-down and subjected to Western blotting followed by exposure to PhosphorImager. $(F)$ Rnf6 targets endogenous LIMK1 for degradation. HeLa cells were transfected with the dominant-negative Myc-Rnf6 $\mathrm{RING}$ expression plasmid. Cells were stained with anti-Myc (green) and anti-LIMK1 (red) antibodies. Note that endogenous LIMK1 levels (red) are greatly increased in cells transfected with Myc-Rnf6 $\Delta$ RING (green).

tions, we detected strong polyubiquitination of LIMK1 using the K48only ubiquitin mutant protein, similar to the ubiquitination pattern detected when wild-type ubiquitin was used (Fig. 4C).

In order to test whether LIMK1 is a physiological target of Rnf6 or RLIM, we cotransfected cells with combinations of LIMK1, RLIM, and Rnf6 expression plasmids (Fig. 4D). We observed that overexpressed Rnf6 induced the degradation of Myc-tagged LIMK1 (Fig. 4D, fifth lane from the left) and that this effect was dependent on a functional RING finger since cotransfection with the Myc-Rnf6 2 RING construct did not reduce LIMK1 levels (Fig. 4D, sixth lane from the left). Addition of the proteasome inhibitor MG132 partially restored LIMK1 levels (Fig. 4D, eighth lane from the left), indicating that Rnf6 targets LIMK1 for proteasomal degradation through the $26 \mathrm{~S}$ proteasome. In these experiments, overexpressed RLIM only marginally targeted LIMK1 for degradation (Fig. 4D, fourth lane from the left).

To further determine if Rnf6 plays a role in the regulation of LIMK1 stability, we analyzed the half-life of LIMK1 protein in the presence or absence of Rnf6 (Fig. $4 \mathrm{E})$. The results obtained in these pulse-chase experi- ments demonstrate that GST-LIMK1 is a stable protein with a half-life of $\sim 20 \mathrm{~h}$ (data not shown). However, when coexpressed with Rnf6, the half-life of GST-LIMK1 protein was reduced to only $4 \mathrm{~h}$. Consistent with a role of Rnf6 in ubiquitin-dependent proteasomal degradation, the addition of MG132 increased LIMK1 protein half-life in the presence of Rnf6 overexpression while having no detectable effect on LIMK1 expression in the absence of Rnf6 (Fig. 4E). Although obtained by overexpression experiments, these findings strengthen the notion that Rnf6 is a LIMK1-specific E3 ligase involved in the degradation of LIMK1.

To investigate if Rnf6 is involved in degradation of the endogenous LIMK1 protein, we transfected the RINGfinger-deleted Rnf6 construct that can act in a dominantnegative manner for Rnf6 function, into HeLa, Cos7, or HEK293T cells. As expected, the overexpression of Rnf6 $\Delta$ RING resulted in increased levels of endogenous LIMK1 protein in the transfected cells (Fig. 4F; data not shown), indicating that Rnf6 regulates cellular LIMK concentrations. Combined, these experiments identify Rnf6 as an ubiquitin ligase, able to mediate polyubiquitination of LIMK1, resulting in proteasomal degradation. 
Tursun et al.

\section{Rnf6 and LIMK1 proteins are partially colocalized in primary hippocampal neurons}

To examine whether the interaction between Rnf6 and LIMK1 plays a role during mouse development, we compared the tissue distribution of Rnf6 and LIMK1 by performing immunohistochemistry on mouse embryonic sections. At E12.5-E13, LIMK1 displayed a remarkably similar expression pattern to that of Rnf6 with high expression levels of both proteins in the mouse ventral neural tube as well as in axonal projections of wild-type, erbB3 ${ }^{+/-}$, and erbB3 ${ }^{-/-}$mice (Fig. 5A; data not shown). As previously reported (Foletta et al. 2004) and similar to Rnf6, the diffuse signals indicate that LIMK1 is mainly expressed in the cytoplasm of ventral neural tube neurons.

We next investigated the cellular localization of Rnf6 and LIMK1 by staining cultured mouse primary hippocampal neurons with a specific guinea pig Rnf6 polyclonal antibody and a rat LIMK1 monoclonal antibody. To identify neuronal projections, we used an antibody directed against neurofilament (data not shown). We found that Rnf6 and LIMK1 partially colocalized in cytoplasmic regions around the nuclei as well as in neuronal projections (Fig. 5B,C). Similarly, in growth cones both proteins were expressed in an asymmetric fashion (Fig. 5D). Furthermore, higher magnification revealed that in growth cones, a high degree of overlapping expression consistently occurred only in small areas that appeared as an interface between regions of high Rnf6 or LIMK1 expression (Fig. 5D). This reciprocal protein distribution suggests active regulation of LIMK1 concentrations by Rnf6.

\section{Rnf6 regulates LIMK1 levels in growth cones of primary hippocampal neurons}

Together, the results described above strongly suggest that Rnf6 targets LIMK1 for proteasomal degradation in growth cones. However, several prerequisites must be fulfilled before such a scenario can take place. One prerequisite is that LIMK1 levels must be regulated by the proteasome in growth cones. To test this possibility, we treated primary hippocampal cell cultures with the proteasome inhibitor MG132. Indeed, higher LIMK1 levels were detected in extracts of cells treated with MG132 in a time-dependent manner (Fig. 6A). Focusing on neuronal projections, we found that MG132 treatment of neurons resulted in increased relative LIMK1-fluorescence intensities, indicating increased LIMK1 levels in growth cones (Fig. 6B,C). During the investigated timeframe, this up-regulation was not homogeneous over the entire growth cone but had a more subregional pattern. A mild increase of LIMK1 expression was also observed in the axons upon MG132 treatment. Another prerequisite is that the mouse homolog of $\mathrm{UbcH} 5$, the ubiquitin conjugating enzyme used by Rnf6 to polyubiquitinate LIMK1 (Fig. 4B,C), must be expressed in axonal growth cones. Immunocytochemical staining using a specific antibody directed against $\mathrm{UbcH} 5$ indicated that this enzyme is
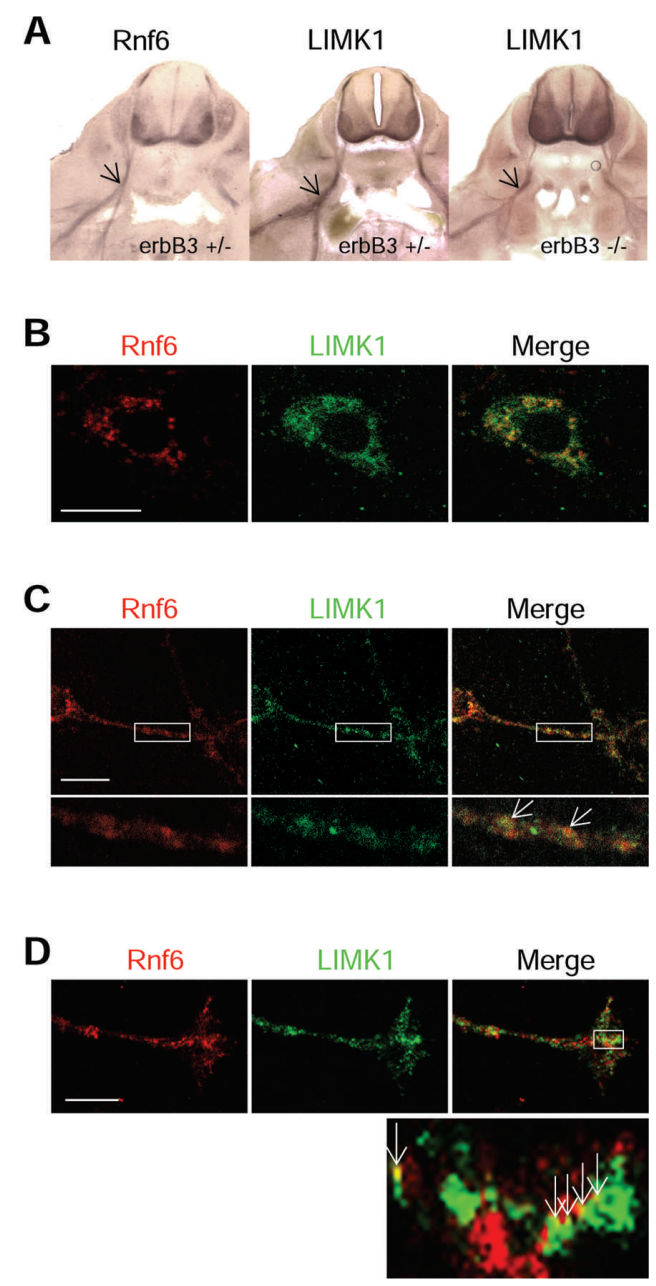

Figure 5. Rnf6 expression partially colocalizes with LIMK1 in neuronal projections. (A) LIMK1 and Rnf6 proteins display an overlapping expression pattern during mouse nervous system development. Vibratome sections of E12.5-E13.5 $\mathrm{erbB3}^{+/-}$ and $\mathrm{erbB}^{-/-}$mice were stained with anti-Rnf6 polyclonal and anti-LIMK1 monoclonal antibodies. Arrows indicate stained axonal projections. (B) Rnf6 and LIMK1 partially colocalize in peri-nuclear punctae of hippocampal neurons. Mouse primary hippocampal neurons were cultured for $4 \mathrm{~d}$ and stained with anti-Rnf6 (red) and rat anti-LIMK1 antibodies (green). The yellow color indicates colocalization of both proteins (merge). Bar, $10 \mu \mathrm{m} .(C)$ Rnf6 and LIMK1 partially colocalize in projections of hippocampal neurons. Mouse primary hippocampal neurons were cultured for $4 \mathrm{~d}$ and stained with antiRnf6 (red) and LIMK1 antibodies (green). Bottom panels show higher magnification of the boxed regions in the top panels. Arrows indicate punctuate regions of colocalization. Bar, $10 \mu \mathrm{m}$. (D) Colocalization of Rnf6 and LIMK1 in growth cones is restricted to a small interface region of the two proteins. Primary hippocampal neurons cultured for $2 \mathrm{~d}$ were stained with anti-Rnf6 (red) and anti-LIMK1 antibodies (green). Neurofilament-positive projections are shown. Bar, $4 \mu \mathrm{m}$. Images were captured by confocal microscopy using a $63 \times$ objective. Enlargement of the boxed region is shown in the lower panel. Note the small area of colocalization as indicated by arrows. 
A

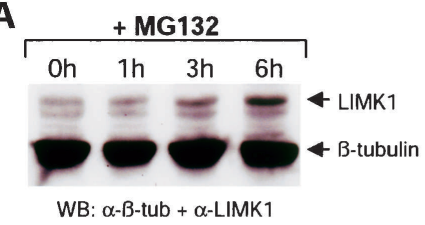

B

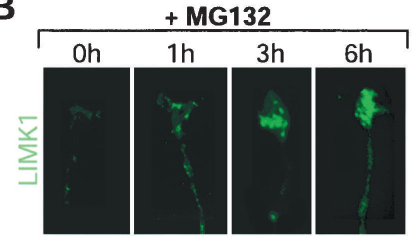

C

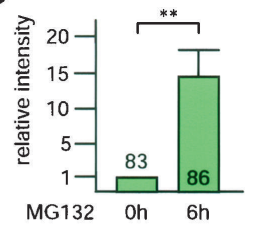

D

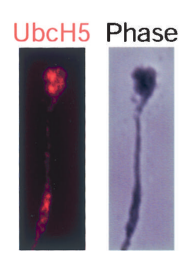

E

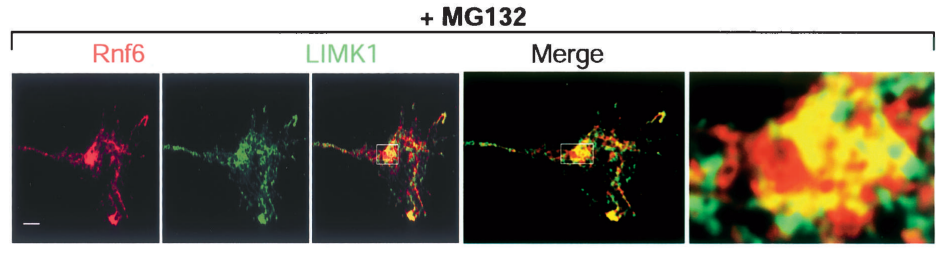

Figure 6. Proteasomal regulation of LIMK1 in growth cones. (A) Proteasomal regulation of LIMK1 levels in cultures of dissociated hippocampal neurons. Western blot analysis of total protein extracts of primary hippocampal neuron cultures treated with the proteasome inhibitor MG132 for 1, 3, and $6 \mathrm{~h}$, probed with anti-LIMK1 and $\beta$-tubulin antibodies. (B) LIMK1 concentrations at the growth cones are regulated by the proteasome. Primary hippocampal neurons cultured for $2 \mathrm{~d}$ were treated for 1,3 , and $6 \mathrm{~h}$ with MG132, followed by staining with anti-LIMK1 antibodies (green). Only neurofilament-positive projections are shown. The timedependent increase in LIMK1 concentrations was so high that the photomultiplier of the confocal microscope was set to a $50 \%$ lower level for cells treated for 3 and $6 \mathrm{~h}$ with MG132. Representative cells from three independent experiments are shown. (C) Relative LIMK1 levels in axonal growth cones of hippocampal neurons after $6 \mathrm{~h}$ of MG132 treatment as measured by fluorescence intensity of same-sized growth cone areas in three independent sets of experiments. Relative fluorescence intensities at $0 \mathrm{~h}$ of MG132 treatment

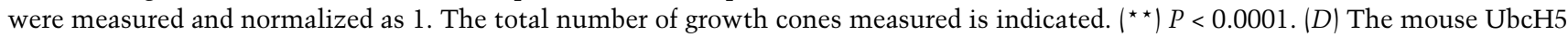
ortholog is expressed in growth cones. Primary hippocampal neurons cultured for $2 \mathrm{~d}$ were stained with anti-UbcH5 antibodies (red). A neurofilament-positive projection is shown. (Right panel) A phase-contrast image of the same neuron. $(E)$ Treatment with proteasome inhibitor results in colocalization of Rnf6 and LIMK1 over large parts of the growth cone. Primary hippocampal neuron cultured for $2 \mathrm{~d}$ and treated with MG132 for $3 \mathrm{~h}$ were stained for Rnf6 (red) and LIMK1 (green). Only neurofilament-positive projections are shown. Bar, $4 \mu \mathrm{m}$. Images were captured by confocal microscopy using a $63 \times$ objective. Enlargement of the boxed region is shown in the right panel. Note that colocalization of Rnf6 and LIMK1 occurs over large parts of the growth cone. Similar results were obtained in five independent experiments.

expressed throughout cultured hippocampal neurons including growth cones (Fig. 6D; data not shown). To further investigate whether the proteasome and Rnf6 are involved in the turnover of LIMK1 in axonal growth cones, we treated primary hippocampal neuron cultures with proteasome inhibitor and examined the overlap in the expression of Rnf6 and LIMK1. Immunocytochemistry of MG132-treated cells revealed that the colocalization of LIMK1 with Rnf6 in growth cones was notably increased in comparison to untreated cells (cf. Figs. 5D and $6 \mathrm{E})$. These experiments were repeated five times with consistent results showing that growth cone regions expressing high levels of Rnf6 and LIMK1 were largely overlapping in cells treated with MG132, indicating that the potential for both proteins to localize in growth cone subregions is similar.

To demonstrate that Rnf6 targets LIMK1 for degradation in growth cones, we investigated LIMK1 levels in primary hippocampal neuron cultures treated with Rnf6 siRNA. Indeed, the down-regulation of Rnf6 resulted in increased overall LIMK1 levels (Fig. 7A). Immunocytochemical staining of Rnf6-siRNA-treated primary hippocampal neurons showed that the down-regulation of Rnf6 was accompanied by increased LIMK1 expression in growth cones (Fig. 7B). To provide even further evidence that Rnf6 targets LIMK1 for degradation in growth cones, we overexpressed a GFP-Rnf6 fusion protein in primary hippocampal neurons. Whereas overexpressed
GFP alone showed colocalization with endogenous LIMK1 over wide areas in the growth cones, there was little colocalization of GFP-Rnf6 with LIMK1 (Fig. 7C, top two panels). In contrast, incubation of the GFP-Rnf6 transfected neurons with the proteasome inhibitor MG132 for $2 \mathrm{~h}$ dramatically increased the colocalization of both proteins (Fig. 7C, third panel). Furthermore, overexpression of the dominant-negative GFP-Rnf6 2 RING protein resulted in its strong colocalization with LIMK1 (Fig. 7C, lower panel), similar to that observed after treatment of Rnf6-transfected neurons with MG132. Together, these results show not only that LIMK1 levels are regulated by the proteasome in neuronal growth cones, but also indicate that Rnf6 participates actively in targeting LIMK1 for proteasomal degradation in this structure.

\section{Opposing roles of Rnf6 and LIMK1 during axon outgrowth}

It has previously been reported that the overexpression of LIMK1 in primary hippocampal neurons results in increased length of early neurites (Rosso et al. 2004). Therefore, we investigated if LIMK1 and Rnf6 are functionally linked during axon outgrowth using RNAi. We first tested the efficiency of our LIMK1 siRNA and showed that it greatly reduced the level of LIMK1 in cultures of primary hippocampal neurons (Fig. 8A). Next, 
Tursun et al.

Figure 7. Rnf6 regulates LIMK1 levels in axonal growth cones. (A) Western blot of total protein extracts of primary hippocampal neuron cultures transfected with Rnf6 siRNA and control siRNA (c-Rnf6 siRNA) was probed with Rnf6 antibodies. The same blot was reprobed with LIMK1 antibodies and, as a loading control, with RLIM antibodies. Note that in cells transfected with Rnf6 siRNA, Rnf6 levels are decreased while LIMK1 levels are increased when compared with control-transfected cell extracts. Transfection efficiencies were $\sim 70 \%$. (B) Representative growth cones of primary hippocampal neurons cotransfected with control siRNA or Rnf6 siRNA, together with the GFP-C3 expression vector to visualize transfected neurons. Cells were stained with Rnf6 (red) and LIMK1 (blue) antibodies. Bar, $6 \mu \mathrm{m}$. Note that in Rnf6 siRNA-transfected cells Rnf6 levels decrease, whereas LIMK1 levels increase. $(C)$ Overexpressed GFP-Rnf6 does not colocalize with endogenous LIMK1 in growth cones. Primary hippocampal neurons were transfected with GFP-Rnf6, GFP-Rnf6 $\Delta$ RING, and, as a control, GFP-C3 expression plasmids. GFP is shown in green, LIMK1 in red. Only neurofilament-positive projections are shown. Images were captured by confocal microscopy using a $63 \times$ objective. Enlargement of the boxed regions is shown to the right. Bar $4 \mu \mathrm{m}$. Note that colocalization of GFP and GFP Rnf6 $\Delta$ RING with LIMK1 occurs over large parts of the growth cone, whereas GFP-Rnf6 colocalizes with endogenous LIMK1 only upon inhibition of the proteasome by MG132.

C
A

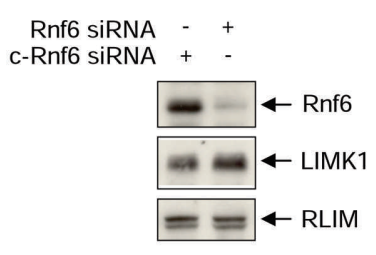

B
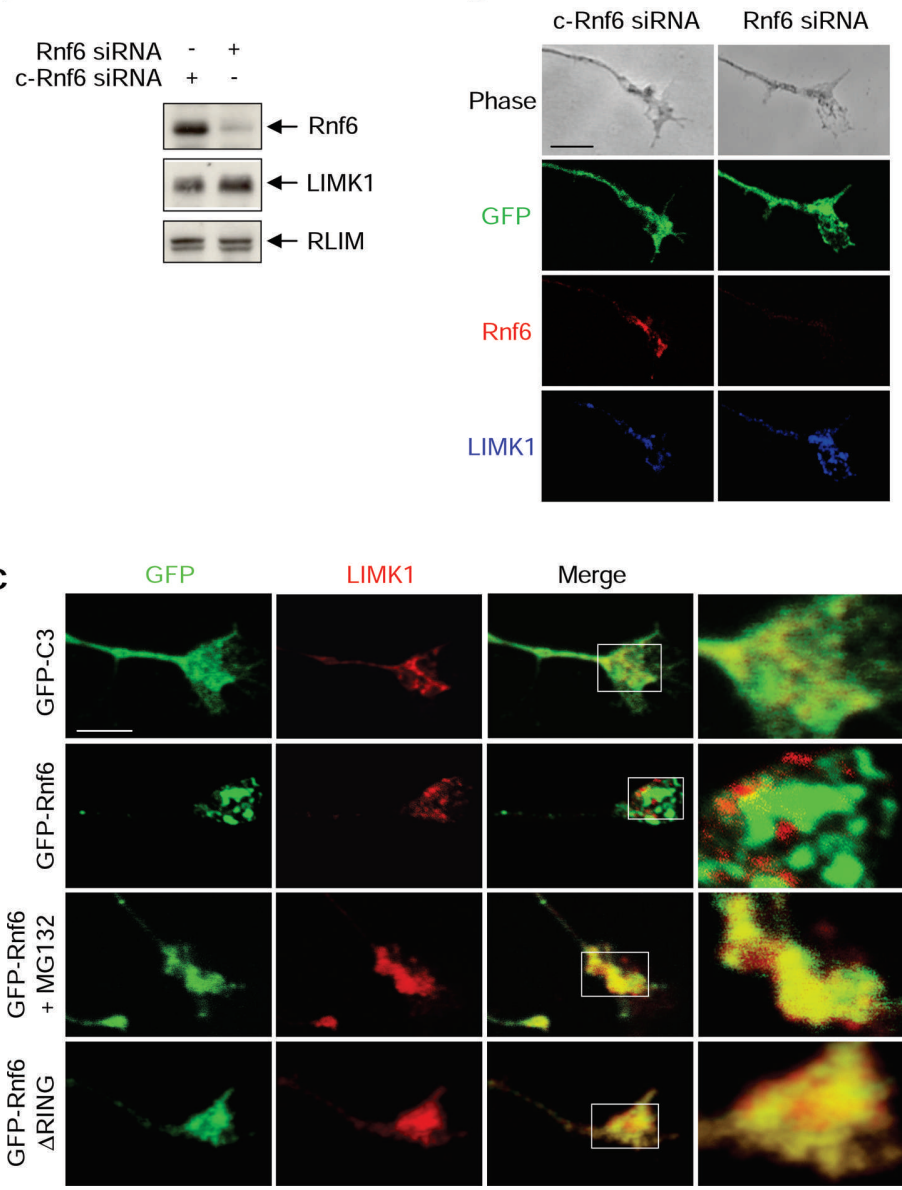

LIMK1

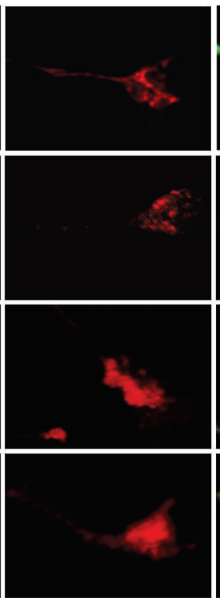

Merge

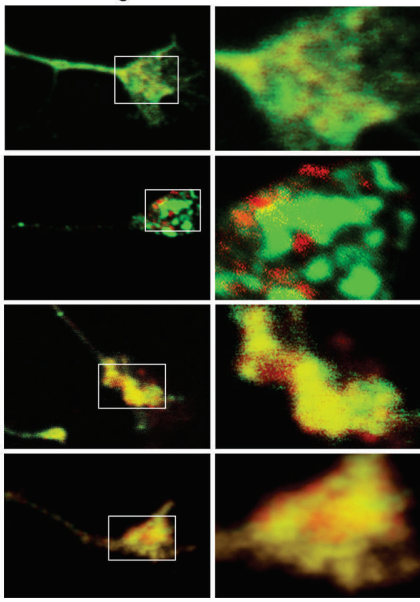

we examined the effect of LIMK1 siRNA on axonal length of the transfected neurons. As expected, neurites of neurons transfected with this siRNA are considerably shorter than those transfected with control siRNA. While the average length of axons treated with control siRNA was $80 \mu \mathrm{m}$, that of neurons expressing LIMK1 siRNA was only $\sim 35 \mu \mathrm{m}$ (Fig. $8 \mathrm{~B}, \mathrm{C}$ ). If the increase in neurite length in Rnf6-siRNA-treated neurons (Fig. $2 \mathrm{C}, \mathrm{D})$ is due to increased LIMK1 levels, then one would expect that, in neurons in which LIMK1 is knocked down, a parallel down-regulation of Rnf6 should have little or no additional effect on axonal length. Indeed, cotransfecting Rnf6-siRNA with LIMK1-siRNA reduced the average length of the Tau-positive neurites axons from $\sim 155 \mu \mathrm{m}$ in neurons treated with Rnf6-siRNA alone to $\sim 47 \mu \mathrm{m}$, a similar length to that of neurons treated with LIMK1 siRNAs (Fig. 8B,C), indicating that the effect of Rnf6 on axon outgrowth is LIMK1dependent. We next investigated whether the observed inhibitory effect of Rnf6 overexpression on neurite outgrowth (Fig. 2E,F) could be rescued by overexpression of LIMK1. In agreement with the above siRNA experiments, the overexpression of GFP-LIMK1 partially restored the Rnf6-mediated down-regulation of average axonal length from $\sim 35 \mu \mathrm{m}$ to $\sim 80 \mu \mathrm{m}$, the length of control-transfected neurons (Fig. 8D,E). These experiments demonstrate that LIMK1 regulates neuronal axons and establish a functional link between Rnf6 and LIMK1 for axonal outgrowth in cultured hippocampal neurons.

\section{Discussion}

The data presented here shed light on the functional role of the ubiquitin-proteasome pathway, with the ubiquitin ligase Rnf6 as an effector, in the regulation of axon outgrowth in cultured hippocampal neurons via the regulation of LIM kinase levels.

Rnf6 is a RING-finger-dependent ubiquitin ligase similar to the previously identified RLIM. The widespread expression of Rnf6 mRNA in a variety of mouse organs suggests that Rnf6 plays a role in many cell types. The overlapping expression patterns of Rnf6 and RLIM in the ventral neural tube suggest that the same cell types express both E3 ligases, indicating potential crossregulation. A high level of Rnf6 protein is found in axonal projections of ventral motor neurons and dorsal root ganglia at E12.5-E13.5, a time point when projections are 


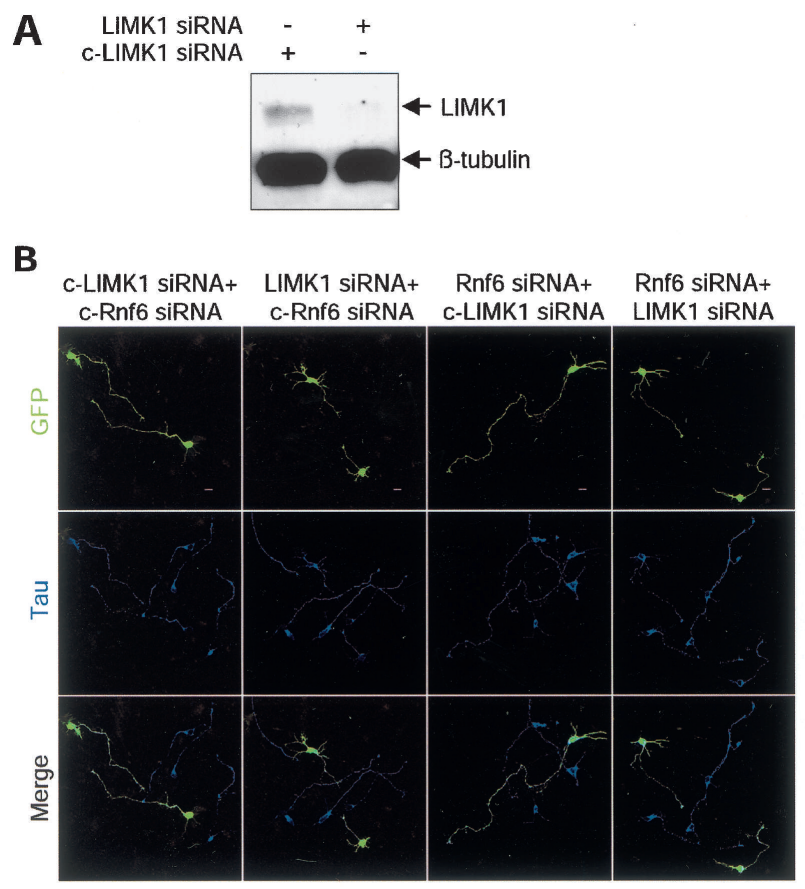

D

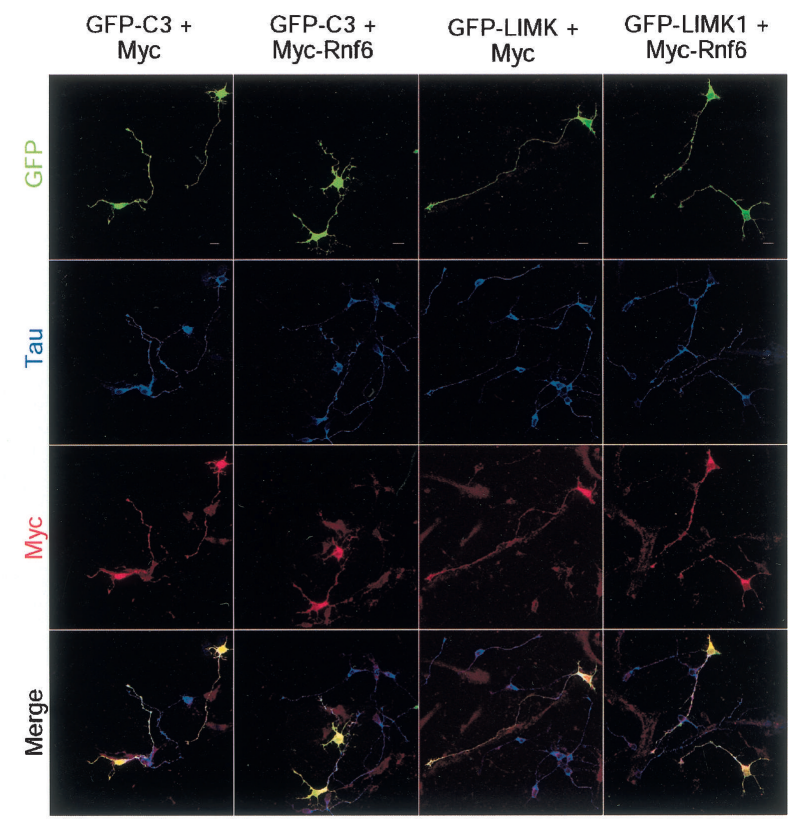

C

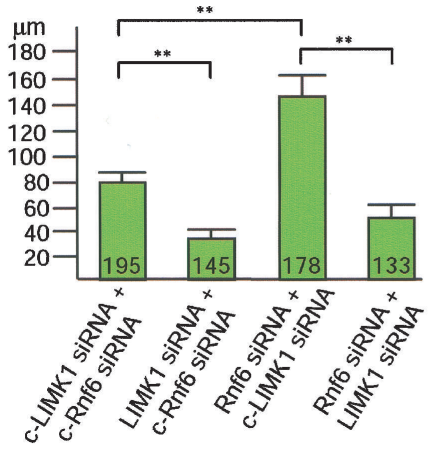

$\mathbf{E}$

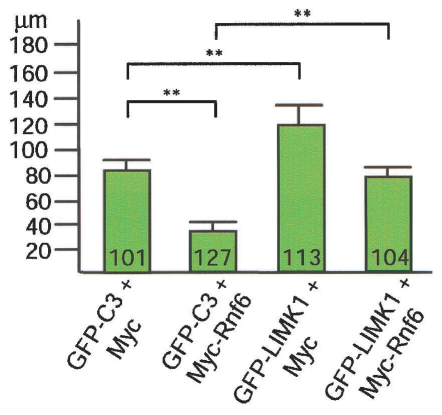

Figure 8. Rnf6 is functionally linked with LIMK1 during axonal growth. (A) Western blot of total protein extracts of primary hippocampal neuron cultures transfected with LIMK1 siRNA or control siRNA (c-LIMK1 siRNA) was probed with anti-LIMK1 mAb and anti- $\beta$-tubulin antibodies as loading controls. Note that in cells transfected with LIMK1 siRNA, the protein levels of LIMK1 are decreased in comparison with control-transfected cell extracts. Transfection efficiencies were $\sim 75 \%$. $(B)$ The increase in axon length mediated by Rnf6 siRNA is dependent on LIMK1. Dissociated primary hippocampal neurons were cultured for $24 \mathrm{~h}$ prior to cotransfection of siRNAs with the empty pEGFP-C3 vector to visualize transfected cells. Twenty-four hours later, cells were stained with anti-Tau antibody. GFP signals (green) and Tau staining (blue) of representative projections are shown. Bars, $10 \mu \mathrm{m}$. Note that the length of axonal projections of neurons cotransfected with both Rnf6 and LIMK1 siRNAs is similar to that of LIMK1 siRNAtransfected neurons. $(C)$ Statistical analysis of axon lengths of neurons cotransfected with Rnf6 and LIMK1 siRNAs. Mean values of three independent experiments are shown. The total number of measured neurons is indicated. Tau-positive projections were measured, using analySIS Pro software. $\left(^{\star \star}\right) P<0.0001$. Error bars indicate SEM. $(D)$ Opposing roles of Rnf6 and LIMK1 for axon outgrowth. Primary neurons were cultured for $24 \mathrm{~h}$ prior to cotransfection of Myc or GFP expression constructs. Twenty-four hours later, cells were processed for immunocytochemistry and stained with anti-Tau and Myc-antibodies. GFP signals (green) and Tau and Myc stainings (blue and red, respectively) of representative projections are shown. Bars, $10 \mu \mathrm{m}$. Note that the length of axonal projections of neurons coexpressing Rnf6 and LIMK1 is similar to control-transfected neurons. (E) Statistical analysis of the lengths of primary hippocampal neurons cotransfected with Rnf6 and LIMK1 expression constructs. Mean values of three independent transfection experiments are shown. The total number of neurons measured is indicated for each transfection. Cells were stained with anti-Myc and anti-neurofilament antibodies. Tau-positive projections were measured using analySIS Pro software. $\left(^{\star \star}\right) P<0.0001$. Error bars indicate SEM.

actively developing, suggesting a role for Rnf6 in the development of these neurons. This is strongly supported by our findings that overexpression of Rnf6 in primary hippocampal neurons leads to a significant decrease in axon length and that the down-regulation of Rnf6 activity by specific siRNAs or dominant-negative Rnf6 stimu- 
lates axon outgrowth (Fig. 2). Since Rnf6 and LIMK1 are also expressed in other neuronal extensions, it is likely that functions of Rnf6 are not restricted only to axonal projections.

We and others have detected LIMK1 expression in growth cones (Endo et al. 2003; Foletta et al. 2004; Rosso et al. 2004). Thus, we compared the expression of LIMK1 with Rnf6 in these structures and found minimal overlapping expression of these two proteins. However, in a relatively small area, resembling an interface between these proteins, colocalization was observed. Since we have shown that endogenous Rnf6 is able to interact with endogenous LIMK1 in cultured neuronal cells (Fig. 3 ), it is most likely that these proteins form complexes in such regions of colocalization. The demonstration that LIMK1 levels are controlled by the proteasome, and that the colocalization of Rnf6 and LIMK 1 in growth cones is dramatically increased upon inhibition of Rnf6 activity by treatment with MG132, shows that both proteins display a similar expression pattern in growth cones and that proteasomal activity is responsible for the observed differences in their cellular localization in untreated cells. These observations are consistent with the notion that Rnf6 actively targets LIMK1 for proteasomal degradation in growth cones. This is further highlighted by our findings that, similarly to neurons transiently overexpressing LIMK1 (Rosso et al. 2004), MG132 treatment also resulted in an increase in growth cone size and the appearance of growth cone-like structures along axons (data not shown). In addition, the result that endogenous LIMK1 colocalizes with overexpressed GFP-Rnf6 in axonal growth cones only upon addition of proteasome inhibitor or with overexpressed dominant-negative RINGfinger-deleted Rnf6, indicates that Rnf6 is directly involved in the turnover of LIMK1 in growth cones. The fact that MG132 treatment induced surprisingly high LIMK1 levels restricted to specific regions of the growth cone further indicates that particularly high LIMK1 turnover takes place in a proteasome-dependent manner in these structures. These results are in agreement with previous findings that attribute rapid local protein degradation to growth cones (Campbell and Holt 2001). Furthermore, our results match well with the finding that actin filaments within axonal growth cones are more dynamic and flexible than those in other neurites (Bradke and Dotti 1999).

Because LIMK1 is important for a large number of cellular activities, its levels must be tightly regulated. One of the ways to regulate cellular LIMK1 activity is by controlling its phosphorylation levels, as its activity correlates with the levels of its phosphorylation. We have recently shown that LIMK1 activity is regulated by slingshot phosphatase, which was first identified as cofilin phosphatase (Soosairajah et al. 2005). Thus, the identification of Rnf6 as an important regulator of LIMK1 levels via proteasomal degradation adds another level of complexity to the regulation of LIMK1 activity.

It is thought that dynamic changes to the cytoskeletal actin network in growth cones play crucial roles for axon growth (Bradke and Dotti 1999). Therefore, it is likely that LIMK1, a key regulator of actin dynamics, plays an important role in the regulation of axonal growth. Indeed, in agreement with previous findings that attribute a role for LIMK1 in axon formation (Rosso et al. 2004), we confirmed that LIMK1 siRNA inhibits axon outgrowth (Fig. 8A,B). A link between Rnf6 and LIMK1 is confirmed by the observation that the stimulating effect on axon outgrowth by Rnf6 siRNA is mostly antagonized upon cotransfection of LIMK1 siRNAs. The incomplete neutralization observed in these experiments is probably due to the fact that our siRNA did not completely remove the respective proteins (Figs. 7A, 8A). The overexpression experiments showed that the Rnf6mediated decrease in axon outgrowth can be rescued by LIMK1 overexpression, further corroborating the functional connection between Rnf6 and LIMK1 (Fig. 8D,E). Combined, our data indicate that the most likely mechanism by which Rnf6 participates in the development of axonal projections is the adjustment of local LIMK1 concentrations necessary for appropriate actin dynamics to occur. In this context, it is interesting to note that inhibitors of proteasome function block responses to guidance cues (Campbell and Holt 2001; Mann et al. 2003). The observations that cues regulating neurite outgrowth and axon guidance are translated into changes in cytoskeletal actin dynamics in growth cones /da Silva and Dotti 2002; Dickson 2002; van Horck et al. 2004; Campbell and Holt 2003) and that the activity of ubiquitin ligases is often regulated (Pickart 2001), open up the intriguing possibility that Rnf6 is involved in axonal pathfinding events by regulating local LIMK1 levels.

As mentioned above, the regulation of LIMK1 levels is of great importance for many cellular functions, and upor down-regulation of its activity may have detrimental effects on a variety of cellular functions. For example, ablation of LIMK1 expression in LIMK1 knockout mice results in abnormalities in dentritic spine morphology and in synaptic function (Meng et al. 2002). In addition, cultured hippocampal neurons from these knockout mice have reduced growth cone size, a very similar phenotype to that shown in this study and in that of Rosso et al. (2004). On the other hand, overexpression of LIMK1 in breast or prostate cancer cell lines increases their invasiveness, implicating LIMK1 in cancer metastasis (Davila et al. 2003; Yoshioka et al. 2003). The fact that LIMK1 has been involved in regulatory events such as the dynamics of the Golgi apparatus as well as cell motility and the development of hippocampal dentritic spines, synapses, and LTP (Sarmiere and Bamburg 2002; Fukazawa et al. 2003; Gungabissoon and Bamburg 2003; Rosso et al. 2004) further indicates the importance of tight regulation of the level and activity of this protein.

There are several lines of evidence that point toward a more general involvement of Rnf6 in LIMK regulation. We have shown that Rnf6 is able to target LIMK1 for degradation in a variety of different cell types, including HeLa, HEK293T, Cos7, and primary hippocampal neurons. Moreover, the cytoplasmic expression of Rnf6 and its partial colocalization with LIMK1 in many organs and cellular compartments (data not shown) make it 
likely that Rnf6 might also affect functions mediated by LIMK1 in cellular compartments and cell types other than neuronal growth cones. Thus, our findings strongly suggest a more general participation of Rnf6 in the regulation of LIMK1 levels.

It has been speculated that ubiquitin ligases are critical regulators of proteins important for the integration/ synchronization of specific cellular programs (Conaway et al. 2002; Bach and Ostendorff 2003). We and others have demonstrated that Rnf6 and RLIM ubiquitin ligases control the cellular concentrations of key proteins such as CLIM (Ostendorff et al. 2002; Hiratani et al. 2003), HDAC (Kramer et al. 2003), and LIMK. This is of particular interest, as Rnf6 and RLIM (Scanlan et al. 1999; Lo et al. 2002), as well as their substrate proteins LMO (Rabbitts et al. 1999; Visvader et al. 2001; Wang et al. 2004), LIMK1 (Yoshioka et al. 2003), and CLIM (Matthews and Visvader 2003), are associated with biological processes involved in human pathology such as impaired embryonic development, metastasis, and invasion of cancer. Since many of these proteins are also involved in neuronal differentiation and the development of proper neuronal projections (Becker et al. 2002; Meng et al. 2002, 2003; Thaler et al. 2002), these results indicate that Rnf6 and RLIM not only play central roles in coordinating neuronal development but may also be involved in oncogenesis.

In summary, we propose that the ubiquitin ligase Rnf6 mediates proteasomal regulation of local LIMK1 levels, thereby playing a central role in controlling actin dynamics in subcellular structures.

\section{Materials and methods}

Cloning of Rnf6, plasmids, and antibodies

RT-PCR was used to isolate full-length cDNAs encoding mouse and human Rnf6 as described previously (Ostendorff et al. 2000) on mRNA prepared from whole E9.5 mouse embryos and HeLa cells. Amplified cDNAs were sequenced and subcloned in various bacterial and eukaryotic expression vectors. The plasmids used in this work were full-length GFP-Rnf6, GFP-Rnf6 $\Delta$ RING ( $\Delta 589-667)$, and GFP-LIMK1 in pEGFP-C3 (Clontech); and fulllength Myc-tagged Rnf6, Rnf6 $\Delta$ RING, and RLIM $\Delta$ RING in pCS2-MT. The mammalian GST-LIMK1 expression construct was previously described (Foletta et al. 2003).

Rnf6 polyclonal antibodies were raised in guinea pigs against a region of the mouse protein that displays little sequence homology with RLIM (amino acids 225-362) for highest specificity. Previously described antibodies against mouse RLIM (Bach et al. 1997; Ostendorff et al. 2002) were used for protein detection in Western blots, immunocytochemistry, and immunohistochemistry. Mouse anti-LIMK1 monoclonal antibody (mAb; Transduction Laboratories) and the rat anti-LIMK1 mAb (Foletta et al. 2004) were used for Western blots and for immunocyto- and histochemistry, respectively. Primary hippocampal neurons were stained with neurofilament $200 \mathrm{mAb}$ (Sigma), Tau (DakoCytomation), and UbcH5 antibody (Chemicon). Anti-Myc polyclonal (Santa Cruz) and 9E10 mAb (Roche) and anti-HA.11 $\mathrm{mAb}(\mathrm{BabCo})$ were used to detect and immunoprecipitate $\mathrm{M}$ yc- and HA-tagged proteins, respectively. As loading control for Western blots, the $\beta$-tubulin (Covance) and GFP (abcam) antibodies were used. Secondary antibodies were horseradish peroxidase-anti-mouse and horseradish peroxidase-protein A (Bio-Rad). Biotinylated secondary antibodies for the immunohistochemistry were goat anti-guinea pig (Vector) and goat antirat (MoBiTec). For immunocytochemistry, we used Alexa 488 goat anti-mouse (MoBiTec), Alexa 488 goat anti-guinea pig (MoBiTec), Cy3 goat anti-rabbit (Jackson), Cy5 goat anti-rabbit (MoBiTec), and Cy5 goat anti-rat (MoBiTec).

Transient transfections, immunocytochemistry, immunohistochemistry, and in vitro ubiquitination assays

The cell lines used in this work were CHO, Cos7, HEK293T, and HeLa. Transient transfections and cotransfections of cell lines and immunocytochemical experiments were carried out as described previously (Ostendorff et al. 2002). Fluorescent images were taken and analyzed on a confocal microscope (Leica SP2). Immunohistochemical staining was performed on E12.5 mouse embryo sections. Briefly, embryos were fixed in $4 \%$ paraformaldehyde for $2 \mathrm{~h}$, then de- and rehydrated in EtOH. Endogenous peroxidases were quenched with $0.3 \% \mathrm{H}_{2} \mathrm{O}_{2}$ for $30 \mathrm{~min}$ in PBS. After washing, the sections were blocked in $10 \%$ bovine serum and $0.2 \%$ BSA followed by incubation with the primary antibody. Following additional washings, the sections were incubated with the secondary antibody. DAB staining was performed using the Vectastain $\mathrm{ABC}$ Kit (Vector) according to the manufacturer's instructions. The Rnf6- and RLIM-dependent in vitro ubiquitination reactions were carried out as described previously (Ostendorff et al. 2002) using mutant ubiquitin proteins $\mathrm{K} 0$ and K48only (Boston Biochem).

\section{GST pull-down and coimmunoprecipitations}

The in vitro protein-protein interaction experiments were carried out with bacterially expressed GST fusion proteins and ${ }^{35} \mathrm{~S}$-labeled proteins produced using the TNT in vitro transcription/translation kit (Promega) as described (Ostendorff et al. 2002). For coimmunoprecipitations, HEK293T cells were cotransfected with HA-tagged LIMK1 and either Myc-tagged Rnf6 $\Delta$ RING or Myc-tagged RLIM $\Delta$ RING. Cells were lysed in IP-buffer (50 mM Tris at pH 7.6, $150 \mathrm{mM} \mathrm{NaCl}, 1 \mathrm{mM}$ EDTA, $0.1 \%$ Nonidet P-40, 5\% glycerol, protease inhibitors) and clarified by centrifugation at $4^{\circ} \mathrm{C}$. Lysates were incubated with corresponding antibodies for $2 \mathrm{~h}$ at $4^{\circ} \mathrm{C}$ after 30 min of preclearing with protein-A/G agarose beads (Amersham). Beads were washed $(50 \mathrm{mM}$ Tris at $\mathrm{pH} 7.6,150 \mathrm{mM} \mathrm{NaCl}, 1 \mathrm{mM}$ EDTA, $0.1 \%$ Nonidet P- 40 , protease inhibitors) at $4^{\circ} \mathrm{C}$. Immunoprecipitated proteins were analyzed by SDS-PAGE followed by Western blotting. The membrane was probed with the indicated primary antibodies followed by incubation with anti-HA mouse $\operatorname{IgG}(\mathrm{BabCo})$ and horseradish peroxidase-conjugated anti-mouse antibody (Sigma). Coimmunoprecipitations of endogenous proteins were performed alike on protein extracts prepared from cultured hippocampal neurons.

\section{Half-life experiments}

For the half-life experiments, GST-LIMK1 cDNA was transfected with or without RNF6 cDNA into Cos7 cells by electroporation. Forty-eight hours post-transfection, the cells were washed twice with methionine-free DME medium containing $1 \%$ FCS. The cells were then incubated for $1 \mathrm{~h}$ in the same medium containing $0.1 \mathrm{mCi} / \mathrm{mL}^{35} \mathrm{~S}$-methionine to label all the proteins in the transfected cells. After two washes with normal culture media (DME/10\% FCS), the cells were incubated in the same media with or without $15 \mathrm{mM}$ of the Proteosome inhibitor 
MG132 (Sigma) for various times. At each time point, cells were harvested, lysed in protein lysis buffer $(50 \mathrm{mM}$ Tris at $\mathrm{pH} 7.4$, $150 \mathrm{mM} \mathrm{NaCl}, 1 \%$ Triton X-100, and protease inhibitor cocktail tablet [Roche]), and sonicated for $10 \mathrm{sec}$. Cell debris was removed by centrifugation at 13,000 rpm for $10 \mathrm{~min}$. GST-LIMK1 was pulled-down with glutathione-Sepharose beads. Pulleddown proteins were resolved on $10 \%$ Tris-Glycine precast polyacrylamide gel followed by transfer to nitrocellulose filter, and the membrane was analyzed by exposure to PhosphorImager.

\section{Primary hippocampal neuronal cultures}

Primary cultures of hippocampal neurons were prepared from adult mice as reported previously (Neuhoff et al. 1999). For axonal outgrowth assays, transient transfections of primary hippocampal neurons were carried on cells cultured for $24 \mathrm{~h}$. All transfections were performed using Lipofectamin 2000 (Invitrogen) according to the manufacturer's instructions. Primary hippocampal neurons were fixed and stained $24 \mathrm{~h}$ after transfection. Proteasome inhibitor was added to transfected cells $2 \mathrm{~h}$ prior to fixation. A single neurofilament/Tau-positive projection was considered an axon when its length was at least twice as long as all the other projections. Axon lengths of transfected neurons were determined using the analySIS Pro software (Soft Imaging System $\mathrm{GmbH}$ ). Only the longest, neurofilament/Taupositive projection of each transfected neuron was measured. For each point, three independent transfections were carried out. The error bars reflect SEM of the mean. Statistical significance was calculated according to the Student's $t$-test. The fluorescence intensity of same-sized growth cone areas was quantified with the Leica Confocal Software, measured in three independent sets of experiments. To prevent bleaching of GFP (green channel) and Cy3 (red channel) signals, pictures were focused using Cy5 signals (blue channel) before image recording. Photomultiplier settings were predetermined to avoid pixel saturation. Images of projections were taken on a confocal microscope (Leica SP2) using the $63 \times$ objective, and enlargements were obtained by digital image processing. The sequences of siRNAs (STEALTH; Invitrogen) used in this study were as follows: Rnf6 siRNA, UUUCUGAGUCUCCAUCACUUGCCGC (antisense); c-Rnf6 siRNA, UUUCGCGAGUCUCUCUACUUCACGC (antisense); LIMK1 siRNA, UAGUACUGGUGUGAAAGGGAGA CGC (antisense); and c-LIMK1 siRNA, UAGGAAUGUCGUGAG UGAAAGGCGC. Cotransfections of these siRNAs were carried out with the empty pEGFP-C3 vector.

\section{ACKNOWLEDGMENTS}

We are grateful to I. Hermans-Borgmeyer and T. Hoppe for critically reading the manuscript; R. Assoian, G.N. Gill, and K. Mizuno for plasmids; M. Miehe and D. Riethmacher for the Erb3 ${ }^{-/-}$ embryos; and M. Braun and S. Siegel for technical assistance. B.T. was a doctoral fellow of the Boehringer Ingelheim Foundation. S.A.J. was supported by the Alexander von Humboldt Foundation and a post-doctoral fellowship from the National Institutes of Health (1F32CA108324). I.B. was supported by a Heisenberg scholarship of the Deutsche Forschungsgemeinschaft (DFG) and the Chica and Heinz Schaller Foundation. This work was funded by grants from the DFG to I.B. and the Australian NHMRC to O.B.

\section{References}

Arber, S., Barbayannis, F.A., Hanser, H., Schneider, C., Stanyon, C.A., Bernard, O., and Caroni, P. 1998. Regulation of actin dynamics through phosphorylation of cofilin by LIM-kinase. Nature 393: 805-809.
Bach, I. 2000. The LIM domain: Regulation by association. Mech. Dev. 91: 5-17.

Bach, I. and Ostendorff, H.P. 2003. Orchestrating nuclear functions: Ubiquitin sets the rhythm. Trends Biochem. Sci. 28: 189-195.

Bach, I., Carriere, C., Ostendorff, H.P., Andersen, B., and Rosenfeld, M.G. 1997. A family of LIM domain-associated cofactors confer transcriptional synergism between LIM and Otx homeodomain proteins. Genes \& Dev. 11: 1370-1380.

Bach, I., Rodriguez-Esteban, C., Carriere, C., Bhushan, A., Krones, A., Rose, D.W., Glass, C.K., Andersen, B., Izpisua Belmonte, J.C., and Rosenfeld, M.G. 1999. RLIM inhibits functional activity of LIM homeodomain transcription factors via recruitment of the histone deacetylase complex. Nat. Genet. 22: 394-399.

Becker, T., Ostendorff, H.P., Bossenz, M., Schlüter, A., Becker, C., Peirano, R.I., and Bach, I. 2002. Multiple functions of LIM domain-binding CLIM/NLI/Ldb cofactors during zebrafish development. Mech. Dev. 117: 75-85.

Bradke, F. and Dotti, C.G. 1999. The role of local actin instability in axon formation. Science 283: 1931-1934.

Campbell, D.S. and Holt, C.E. 2001. Chemotropic responses of retinal growth cones mediated by rapid local protein synthesis and degradation. Neuron 32: 1013-1026.

- 2003. Apoptotic pathway and MAPKs differentially regulate chemotropic responses of retinal growth cones. Neuron 37: 939-952.

Conaway, R.C., Brower, C.S., and Conaway, J.W. 2002. Emerging roles of ubiquitin in transcription regulation. Science 296: $1254-1258$.

Craig, A.M. and Banker, G. 1994. Neuronal polarity. Annu. Rev. Neurosci. 17: 267-310.

da Silva, J.S. and Dotti, C.G. 2002. Breaking the neuronal sphere: Regulation of the actin cytoskeleton in neuritogenesis. Nat. Rev. Neurosci. 3: 694-704.

Davila, M., Frost, A.R., Grizzle, W.E., and Chakrabarti, R. 2003. LIM kinase 1 is essential for the invasive growth of prostate epithelial cells: Implications in prostate cancer. I. Biol. Chem. 278: 36868-36875.

DiAntonio, A. and Hicke, L. 2004. Ubiquitin-dependent regulation of the synapse. Annu. Rev. Neurosci. 27: 223-246.

Dickson, B.J. 2002. Molecular mechanisms of axon guidance. Science 298: 1959-1964.

Dotti, C.G., Sullivan, C.A., and Banker, G.A. 1988. The establishment of polarity by hippocampal neurons in culture. J. Neurosci. 8: 1454-1468.

Endo, M., Ohashi, K., Sasaki, Y., Goshima, Y., Niwa, R., Uemura, T., and Mizuno, K. 2003. Control of growth cone motility and morphology by LIM kinase and Slingshot via phosphorylation and dephosphorylation of cofilin. J. Neurosci. 23: 2527-2537.

Foletta, V.C., Lim, M.A., Soosairajah, J., Kelly, A.P., Stanley, E.G., Shannon, M., He, W., Das, S., Massague, J., and Bernard, O. 2003. Direct signaling by the BMP type II receptor via the cytoskeletal regulator LIMK1. J. Cell Biol. 162: 10891098.

Foletta, V.C., Moussi, N., Sarmiere, P.D., Bamburg, J.R., and Bernard, O. 2004. LIM kinase 1, a key regulator of actin dynamics, is widely expressed in embryonic and adult tissues. Exp. Cell Res. 294: 392-405.

Fukazawa, Y., Saitoh, Y., Ozawa, F., Ohta, Y., Mizuno, K., and Inokuchi, K. 2003. Hippocampal LTP is accompanied by enhanced F-actin content within the dendritic spine that is essential for late LTP maintenance in vivo. Neuron 38: 447460.

Gungabissoon, R.A. and Bamburg, J.R. 2003. Regulation of 
growth cone actin dynamics by ADF/cofilin. J. Histochem. Cytochem. 51: 411-420.

Hiratani, I., Yamamoto, N., Mochizuki, T., Ohmori, S.Y., and Taira, M. 2003. Selective degradation of excess Ldb1 by Rnf12/RLIM confers proper Ldb1 expression levels and Xlim-1/Ldb1 stoichiometry in Xenopus organizer functions. Development 130: 4161-4175.

Hobert, O. and Westphal, H. 2000. Functions of LIM-homeobox genes. Trends Genet. 16: 75-83.

Kramer, O.H., Zhu, P., Ostendorff, H.P., Golebiewski, M., Tiefenbach, J., Peters, M.A., Brill, B., Groner, B., Bach, I., Heinzel, T., et al. 2003. The histone deacetylase inhibitor valproic acid selectively induces proteasomal degradation of HDAC2. EMBO T. 22: 3411-3420.

Lo, H.S., Hu, N., Gere, S., Lu, N., Su, H., Goldstein, A.M., Taylor, P.R., and Lee, M.P. 2002. Identification of somatic mutations of the RNF6 gene in human esophageal squamous cell carcinoma. Cancer Res. 62: 4191-4193.

Lorick, K.L., Jensen, J.P., Fang, S., Ong, A.M., Hatakeyama, S., and Weissman, A.M. 1999. RING fingers mediate ubiquitinconjugating enzyme (E2)-dependent ubiquitination. Proc. Natl. Acad. Sci. 96: 11364-11369.

Mann, F., Miranda, E., Weinl, C., Harmer, E., and Holt, C.E. 2003. B-type Eph receptors and ephrins induce growth cone collapse through distinct intracellular pathways. J. Neurobiol. 57: 323-336.

Matthews, J.M. and Visvader, J.E. 2003. LIM-domain-binding protein 1: A multifunctional cofactor that interacts with diverse proteins. EMBO Rep. 4: 1132-1137.

Meng, Y., Zhang, Y., Tregoubov, V., Janus, C., Cruz, L., Jackson, M., Lu, W.Y., MacDonald, J.F., Wang, J.Y., Falls, D.L., et al. 2002. Abnormal spine morphology and enhanced LTP in LIMK-1 knockout mice. Neuron 35: 121-133.

Meng, Y., Zhang, Y., Tregoubov, V., Falls, D.L., and Jia, Z. 2003. Regulation of spine morphology and synaptic function by LIMK and the actin cytoskeleton. Rev. Neurosci. 14: 233240.

Murphey, R.K. and Godenschwege, T.A. 2002. New roles for ubiquitin in the assembly and function of neuronal circuits. Neuron 36: 5-8.

Neuhoff, H., Roeper, J., and Schweizer, M. 1999. Activitydependent formation of perforated synapses in cultured hippocampal neurons. Eur. J. Neurosci. 11: 4241-4250.

Ostendorff, H.P., Bossenz, M., Mincheva, A., Copeland, N.G., Gilbert, D.J., Jenkins, N.A., Lichter, P., and Bach, I. 2000. Functional characterization of the gene encoding RLIM, the corepressor of LIM homeodomain factors. Genomics 69: $120-130$.

Ostendorff, H.P., Peirano, R.I., Peters, M.A., Schlüter, A., Bossenz, M., Scheffner, M., and Bach, I. 2002. Ubiquitination-dependent cofactor exchange on LIM homeodomain transcription factors. Nature 416: 99-103.

Pickart, C.M. 2001. Mechanisms underlying ubiquitination. Annu. Rev. Biochem. 70: 503-533.

Rabbitts, T.H., Bucher, K., Chung, G., Grutz, G., Warren, A., and Yamada, Y. 1999. The effect of chromosomal translocations in acute leukemias: The LMO2 paradigm in transcription and development. Cancer Res. 59: 1794s-1798s.

Riethmacher, D., Sonnenberg-Riethmacher, E., Brinkmann, V., Yamaai, T., Lewin, G.R., and Birchmeier, C. 1997. Severe neuropathies in mice with targeted mutations in the ErbB3 receptor. Nature 389: 725-730.

Rosso, S., Bollati, F., Bisbal, M., Peretti, D., Sumi, T., Nakamura, T., Quiroga, S., Ferreira, A., and Caceres, A. 2004. LIMK1 regulates Golgi dynamics, traffic of Golgi-derived vesicles, and process extension in primary cultured neurons.
Mol. Biol. Cell 15: 3433-3449.

Sanes, J.R. and Lichtman, J.W. 1999. Development of the vertebrate neuromuscular junction. Annu. Rev. Neurosci. 22: 389-442.

Sarmiere, P.D. and Bamburg, J.R. 2002. Head, neck, and spines: A role for LIMK-1 in the hippocampus. Neuron 35: 3-5.

Scanlan, M.J., Gordan, J.D., Williamson, B., Stockert, E., Bander, N.H., Jongeneel, V., Gure, A.O., Jager, D., Jager, E., Knuth, A., et al. 1999. Antigens recognized by autologous antibody in patients with renal-cell carcinoma. Int. J. Cancer 83: 456464.

Shi, S.H., Jan, L.Y., and Jan, Y.N. 2003. Hippocampal neuronal polarity specified by spatially localized $\mathrm{mPar} 3 / \mathrm{mPar} 6$ and PI 3-kinase activity. Cell 112: 63-75.

Shirasaki, R. and Pfaff, S.L. 2002. Transcriptional codes and the control of neuronal identity. Annu. Rev. Neurosci. 25: 251281.

Soosairajah, J., Maiti, S., Wiggan, O., Sarmiere, P., Moussi, N., Sarcevic, B., Sampath, R., Bamburg, J.R., and Bernard, O. 2005. Interplay between components of a novel LIM kinaseslingshot phosphatase complex regulates cofilin. EMBO $J$. 24: 473-486.

Thaler, J.P., Lee, S.K., Jurata, L.W., Gill, G.N., and Pfaff, S.L. 2002. LIM factor Lhx3 contributes to the specification of motor neuron and interneuron identity through cell-typespecific protein-protein interactions. Cell 110: 237-249.

van Horck, F.P., Weinl, C., and Holt, C.E. 2004. Retinal axon guidance: Novel mechanisms for steering. Curr. Opin. Neurobiol. 14: 61-66.

Visvader, J.E., Venter, D., Hahm, K., Santamaria, M., Sum, E.Y., O'Reilly, L., White, D., Williams, R., Armes, J., and Lindeman, G.J. 2001. The LIM domain gene LMO4 inhibits differentiation of mammary epithelial cells in vitro and is overexpressed in breast cancer. Proc. Natl. Acad. Sci. 98: 1445214457.

Wang, N., Kudryavtseva, E., Ch'en, I.L., McCormick, J., Sugihara, T.M., Ruiz, R., and Andersen, B. 2004. Expression of an engrailed-LMO4 fusion protein in mammary epithelial cells inhibits mammary gland development in mice. Oncogene 23: $1507-1513$.

Weissman, A.M. 2001. Themes and variations on ubiquitylation. Nat. Rev. Mol. Cell Biol. 2: 169-178.

Yang, N., Higuchi, O., Ohashi, K., Nagata, K., Wada, A., Kangawa, K., Nishida, E., and Mizuno, K. 1998. Cofilin phosphorylation by LIM-kinase 1 and its role in Rac-mediated actin reorganization. Nature 393: 809-812.

Yoshioka, K., Foletta, V., Bernard, O., and Itoh, K. 2003. A role for LIM kinase in cancer invasion. Proc. Natl. Acad. Sci. 100: $7247-7252$. 


\section{Erratum}

Genes \& Development 19: 2307-2319 (2005)

The ubiquitin ligase Rnf6 regulates local LIM kinase 1 levels in axonal growth cones Baris Tursun, Anne Schlüter, Marvin A. Peters, Birte Viehweger, Heather P. Ostendorff, Juliana Soosairajah, Alexander Drung, Michael Bossenz, Steven A. Johnsen, Michaela Schweizer, Ora Bernard, and Ingolf Bach

In the above-mentioned paper, there was an error in the reproduction of Figure $6 \mathrm{E}$ in which one panel was mistakenly duplicated. The correct figure appears below, with its legend.

A

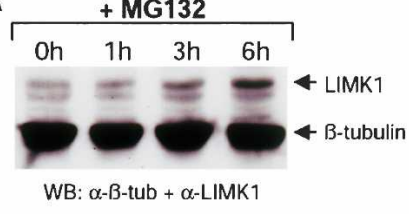

B

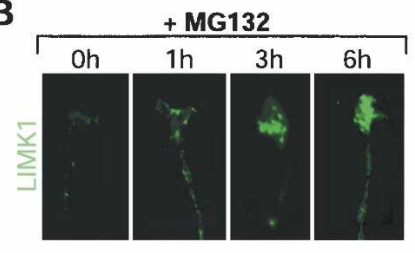

C

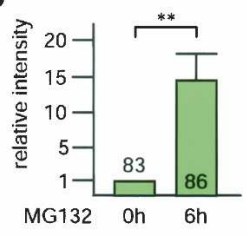

D

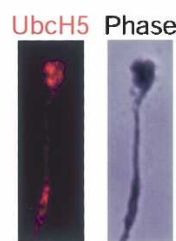

$E$

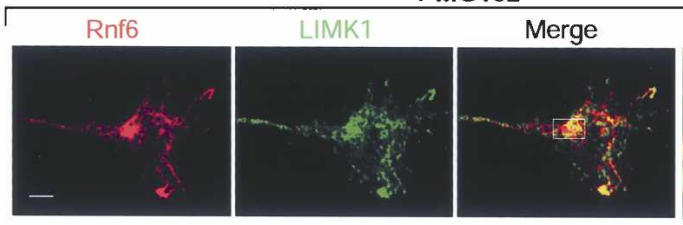

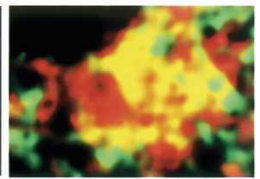

Figure 6. Proteasomal regulation of LIMK1 in growth cones. (A) Proteasomal regulation of LIMK1 levels in cultures of dissociated hippocampal neurons. Western blot analysis of total protein extracts of primary hippocampal neuron cultures treated with the proteasome inhibitor MG132 for 1, 3, and $6 \mathrm{~h}$, probed with anti-LIMK1 and $\beta$-tubulin antibodies. (B) LIMK1 concentrations at the growth cones are regulated by the proteasome. Primary hippocampal neurons cultured for $2 \mathrm{~d}$ were treated for 1,3 , and $6 \mathrm{~h}$ with MG132, followed by staining with anti-LIMK1 antibodies (green). Only neurofilament-positive projections are shown. The timedependent increase in LIMK1 concentrations was so high that the photomultiplier of the confocal microscope was set to a $50 \%$ lower level for cells treated for 3 and $6 \mathrm{~h}$ with MG132. Representative cells from three independent experiments are shown. (C) Relative LIMK1 levels in axonal growth cones of hippocampal neurons after $6 \mathrm{~h}$ of MG132 treatment as measured by fluorescence intensity of same-sized growth cone areas in three independent sets of experiments. Relative fluorescence intensities at $0 \mathrm{~h}$ of MG132 treatment were measured and normalized as 1 . The total number of growth cones measured is indicated. $\left(^{\star \star}\right) P<0.0001$. $(D)$ The mouse UbcH5 ortholog is expressed in growth cones. Primary hippocampal neurons cultured for $2 \mathrm{~d}$ were stained with anti-UbcH5 antibodies (red). A neurofilament-positive projection is shown. (Right panel) A phase-contrast image of the same neuron. $(E)$ Treatment with proteasome inhibitor results in colocalization of Rnf6 and LIMK1 over large parts of the growth cone. Primary hippocampal neuron cultured for $2 \mathrm{~d}$ and treated with MG132 for $3 \mathrm{~h}$ were stained for Rnf6 (red) and LIMK1 (green). Only neurofilament-positive projections are shown. Bar, $4 \mu \mathrm{m}$. Images were captured by confocal microscopy using a $63 \times$ objective. Enlargement of the boxed region is shown in the right panel. Note that colocalization of Rnf6 and LIMK1 occurs over large parts of the growth cone. Similar results were obtained in five independent experiments. 


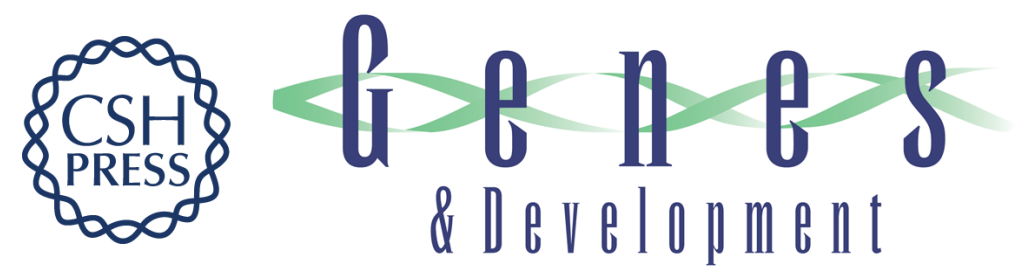

\section{The ubiquitin ligase Rnf6 regulates local LIM kinase 1 levels in axonal growth cones}

Baris Tursun, Anne Schlüter, Marvin A. Peters, et al.

Genes Dev. 2005, 19:

Access the most recent version at doi:10.1101/gad.1340605

Related Content

References

\section{License}

Email Alerting Service
Errata for vol. 19, p. 2307

Genes Dev. November , 2005 19: 2643

This article cites 50 articles, 17 of which can be accessed free at:

http://genesdev.cshlp.org/content/19/19/2307.full.html\#ref-list-1

Articles cited in:

http://genesdev.cshlp.org/content/19/19/2307.full.html\#related-urls

Receive free email alerts when new articles cite this article - sign up in the box at the top right corner of the article or click here.

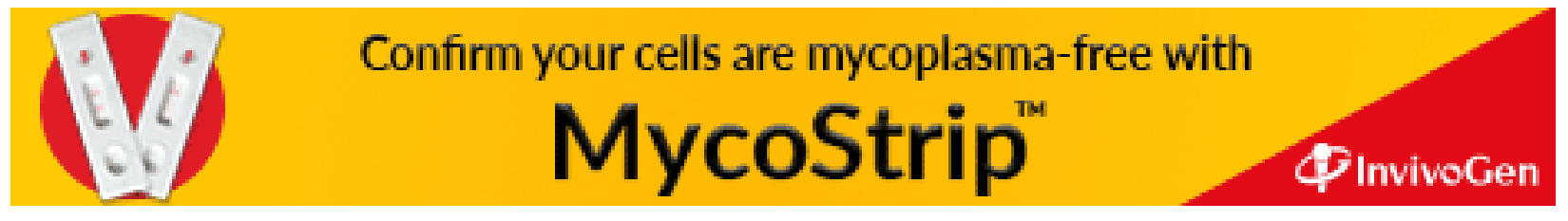

